\title{
Characterizing and Modeling the Structure of Competition Networks*
}

\author{
Bo Yang, Hecheng Wang
}

School of Management, Hangzhou Dianzi University, Hangzhou, China.

Email: yangbo@hdu.edu.cn

Received November $1^{\text {st }}, 2010$; revised December $15^{\text {th }}, 2010$; accepted December $19^{\text {th }}, 2010$.

\begin{abstract}
Not similar with the current interest on collaboration networks research, the focus in this paper is competition networks. The topology of a firm competition network has been investigated empirically and theoretically. We have found that four fundamental characteristics emerge simultaneously in the competition network, including hierarchical modularity, positive degree correlation, power-law degree distribution and self-similarity. The theoretical model we proposed can predict these structural patterns successfully. The obtained results are significant for further network analysis of the omnipresent competitive phenomena.
\end{abstract}

Keywords: Firm Competition, Structural Embeddedness, Complex Networks

\section{Introduction}

The last few years have witnessed a tremendous activity devoted to the characterization and understanding of networked systems. The focus is shifting away from the analysis of single small networks and the properties of individual vertex or edges to consideration of large-scale statistical properties of complex networks [1-3]. Especially, some researches have concentrated on investigating network topology of social and economical systems, using the complex network approach [4-6]. This trend has naturally arisen and been reinforced because many studies have demonstrated that network structure largely affects economical dynamics taking place on the network such as technological progress and diffusion of innovations [7], and plays an important role in the optimization of performance for economical systems [8].

Currently, the concern for network topology of real socio-economical systems mainly focuses on collaboration behaviors. Some examples widely studied involve coauthorship networks [4], strategic partnership alliance [5], supplier-customer networks [6], and etc. In these collaboration networks, any two linked agents cooperate towards their shared aim, for example coauthoring papers and developing new products. Statistical properties of

*Financial support was provided the National Natural Science Foundation of China (No.70872028) and the Development Foundation of Business Administration Discipline of Hangzhou Dianzi University (No ZX100205004-3). this type of networks have been well analyzed.

However, in practice there exists different form of behaviors, i.e. competition behaviors, which are omnipresent in real socio-economical systems, such as firms competing for market share, employees competing for promotion, and etc. Two linked agents in competition networks compete but not collaborate towards their respective aims. Current literature have provided little knowledge about the topology of competition networks, which are worthy of our concern. However, in the field of sociology, two valuable papers [9] and [10] give us insight into the question how to study the competitive behaviors under network circumstances. The common point of these two papers is that they adopt a structural embeddedness perspective [11], which suggests that competitors are embedded in a network of relationship that influences their competitive behaviors. They argued that the structural embeddedness of competitive dynamics has not been studied explicitly. And they followed the typical network approach, attempting to explore the research question "How does the network of cooperative linkages among competitors influence their competitive behaviors towards each other?" It is certain that the use of the network approach helps advance research in competitive dynamics, and then helps develop a better understanding of competitive phenomena.

Different from the above-mentioned work, the aim of this paper is to investigate the topology of competition 
network empirically and construct a theoretical model to predict the structural patterns. In fact, it has been found that many biological, social and technological empirical networks may share some topological characteristics. The researches in this field early focused on scale-free degree distributions. However, further studies have extended to other properties, such as hierarchical modularity, degree correlation and self-similarity, which have been demonstrated to be fundamental and significant for dynamic processes taking place on networks. Although these characteristics are of much significance, more empirical proofs in the further research should be found to support their universality. In this paper, we will see that the empirical firm competition network discussed below exhibits power-law degree distribution, which has been widely studied in current literature. Meanwhile, other three characteristics are found to emerge in this firm competition network, also.

On the other hand, motivated by empirical analysis, research on theoretical network models to predict the empirically-observed structural patterns has attracted a large interest. There are Barabási-Albert model exhibiting scale-free degree distribution [12], hierarchical network model displaying scale-free and a high degree of clustering [13], connecting nearest-neighbor model that appears to display multiple features including scale-free degree distribution, hierarchical modularity and positive degree correlation [14], etc. In summary, the focus of model research is shifting away from the analysis of single structural properties to consideration of multiple properties of networks. In this paper, we will also construct a theoretical network model that can predict empirically-observed structural patterns of the competition network successfully.

The outline of this paper is as follows. In Section 2, the topology of an empirically obtained firm competition network is investigated first. Then in Section 3, a theoretical model is proposed. And, in Section 4, we give our conclusions and point to directions for future research.

\section{Empirical Analysis}

\subsection{Description of the Firm Competition Network}

Competition lies everywhere in real socio-economical systems, between from individuals, firms to countries. The attention of this paper is paid on the firm competition network, which is the representative form of competition networks in socio-economical systems. The empirical network is constructed by analyzing data of firms collected in a certain software industrial park in the south of China. Vertices in this network represent firms in the industrial park whose number is 574. Firms in the park develop various application software products by offering technological support for customer needs. Their products involve in the fields of network security, office automation, communications, and etc. In reality, it is known that firms compete by its products including tangible products or intangible services, which accordingly means that the more similar the products are, the more firms compete. According to the above analysis, we can construct the network as follows. For any two firms, if their respective products are similar in function or customer target and accordingly fall in the same product category, there is competitive relationship between them and thus an edge is constructed between these two firms. In the limit of the length, the detail about how to classify the involved products is not stated here. Notice that in the research they have been professionally classified into 51 categories by a group of consultants. Therefore, we obtain an undirected firm competition network that has 6597 edges. The network can be divided into 51 subnetworks, in which all vertices connected to each other obviously, according to the software product categories. There are 479 firms among these 578 firms, which constrain to develop products in a single product category, and thus the vertices representing them belong to one single group only. On the other hand, other firms develop products in several product categories, and thus the vertices representing them belong to several groups simultaneously. The main network measurements often discussed in literature are presented briefly as follows.

1) Network density: The network density is defined as the ratio between the number of actual links and the maximum possible number of links. And the density is a key network-level property that refers to the extent of interconnection among the actors of the network. The network density of this empirical network is 0.04 , sparse to some extent.

2) Vertex degree: The vertex degree is defined as the number of edges connected to this vertex. And the average connectivity of vertices in the network is the mean degree of all vertices. The average connectivity of vertices in this network is 22.99 while the maximum degree is 144, meaning that an individual firm has about 30 competitors in this industrial park on average and there exist some firms which have 144 competitors at most.

3) Mean geodesic distance: According to [1], we use the "harmonic mean" form to calculate this index. The mean geodesic distance of this empirical network is 2.3, which is indeed very small compared with the number of vertices. It is certain that the so-called small world effect exists in this empirical network.

4) Clustering coefficient: The clustering coefficient measures the density of triangles in the network. In another words, it measures the propensity for vertex pairs 
to be connected if they share a mutual neighbor. The clustering coefficient for this network is 0.92 . It has been reported that for social networks, clustering appears to be far greater than non-social networks [15], which can be verified by our case very well.

\subsection{Analysis of Four Structural Properties}

1) Degree distribution: In current literature, there are two forms of degree distribution, power-law form and exponential form, commonly characterizing real networks $[1,2]$. If the distribution would follow power-law form then it would approximately fall on a straight line in a $\log -\log$ plot. Figure 1 shows the cumulative distributions of degrees $P(k)$ for this firm competition network in $\log -\log$ scale. One can see that there is a better fit to the linear behavior in log-log scale and the solid line with slope -2.15 , which indicates that this network appears to exhibit power-law degree distribution.

Reference [12] argued that power-law degree distribution is the consequence of two generic mechanisms. Firstly, networks expand continuously by the addition of new vertices. Secondly, new vertices attach preferentially to sites that are already well-connected. In this firm competition network, the new-added firm vertices will construct competitive relationships with the existing firms in limiting product categories. And obviously the existing high-degree vertices have competitive relationships with many firms in more product categories than low-degree vertices. Therefore, it is reasonable to think that the new-added firms will have higher probability to construct competitive relationship with these high-degree firms than with low-degree firms. It just follows preferential attachment mechanism, and thus power-law degree distribution emerges as a result.

2) Degree correlation: It has been observed that the degrees of adjacent vertices are positively correlated in social networks and negatively correlated in most other networks. Positive correlation is also called assortative mixing that has been proposed to be distinctive feature of social networks [1,15], which means a preference for high-degree vertices to attach to other high-degree vertices, and vice versa. For measuring degree correlation, two quantifying ways are adopted usually in [1], including plotting a one-parameter curve given by $<k_{n n}>$ depending on $\mathrm{k}$, where $<k_{n n}>$ is the average degree of nearest neighbors of vertices with $\mathrm{k}$ links and calculating the Pearson correlation coefficient $r$ of the degrees at either ends of an edge. If the fit to the curve follows $<k_{n n}>\sim k^{v}$ where $v>0$, or Pearson correlation coefficient $r>0$ then the network is characterized by positive degree correlation.

In this paper, we consider both of these measurements. As shown in Figure 2, we could see that $<k_{n n}>$ increases

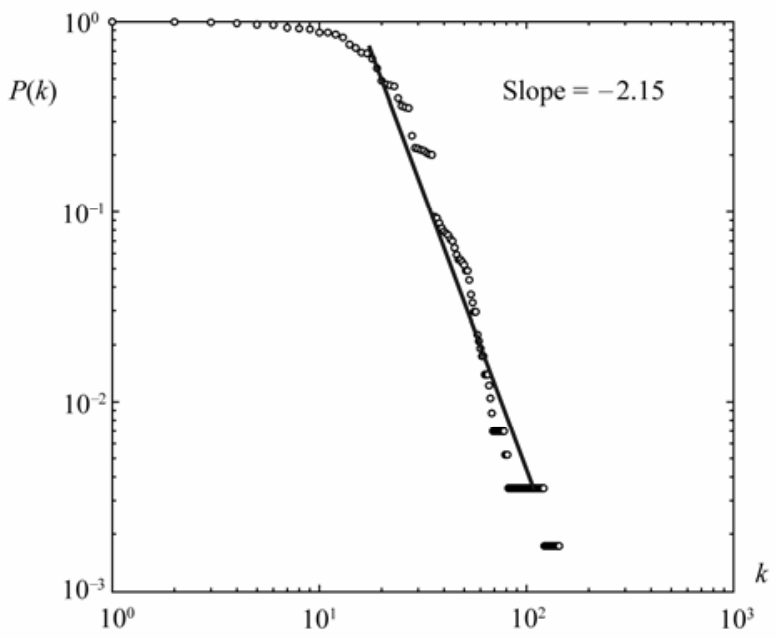

Figure 1. Degree distribution of firm competition network.

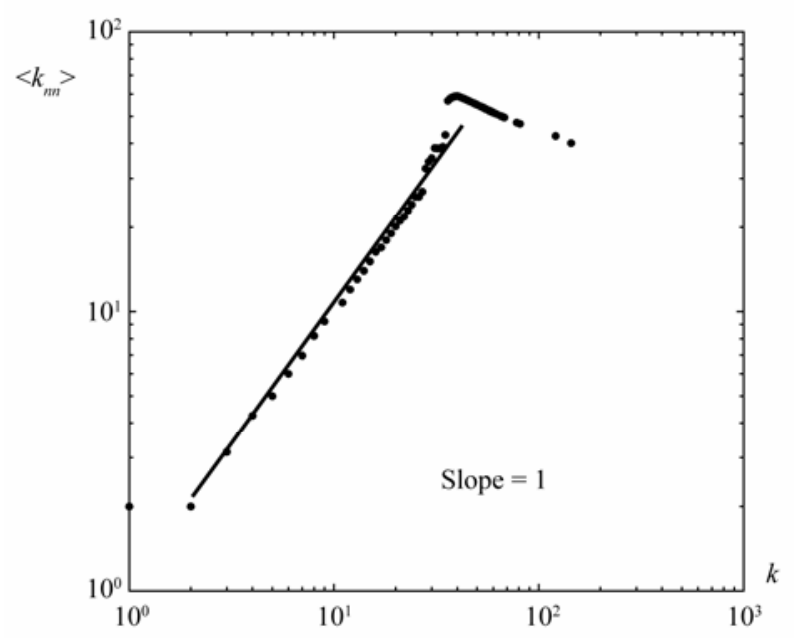

Figure 2. Average degree of nearest neighbors.

with $k$ first, which means that in the cases with small $k$, the average degree of connected neighbors increases with $k$. However, note that $<k_{n n}>$ decreases with $k$ in the tail of the curve, which has also appeared in [14]. We think that the appropriate explanation for this change is that the number of high-degree vertices is very small comparatively. In addition, $r$ is calculated to be $0.4976(>0)$. Therefore, this empirical firm competition network shows positive degree correlation between the degrees of adjacent vertices, which is similar with other social networks.

3) Hierarchical modularity: Also of interest is the hierarchical modularity, which has been found to be shared by some real networks such as actor networks, language networks, World Wide Web, and etc. Indeed, many networks are fundamentally modular [13], as one can easily identify groups of vertices that are highly interconnected 
with each other, but have only a few or no links to vertices outside of the group to which they belong to.

This property is captured by the scaling law $C(k) \sim k^{-\beta}$, where $C(k)$ is the average clustering coefficient of vertices with $k$ links. Figure 3 shows the $C(k)$ curve for this empirical network, while the value of $C(k)$ is in the range of $[0.27,1]$. As the plot indicates, although the obtained $C(k)$ does not follow as closely the scaling law in almost all the range of scale as observed in other networks (see examples presented in [13]), it is clearly evident that there is a linear fit to the real data in log-log scale for values of $k$ between 40 and 144. The slope of solid line in the plot is -1.12 similar with -1 demonstrated to be shown in other real networks. Therefore, it indicates that in this network, many highly interconnected smaller vertices coexist with a few larger vertices, which have lower clustering coefficients, and thus the network exhibits the hierarchical nesting topology. Note that, in cases with $k$ smaller than 40, $C(k)$ seems independent with the increase of $k$. We want to say that the need to satisfy the scaling law in the whole plot is a little strict, and the scale law existing in the tail of the plot is enough to indicate hierarchical modularity [13], just as our case shows.

4) Self-similarity: Recent research papers have proposed that self-similarity is shared by a wide variety of networks, from World Wide Web to cellular networks [16]. This characteristic reflects a power-law relation between the number of boxes needed to cover the network $N_{B}$ and the size of the box $l_{B}$, expressed as $N_{B}\left(l_{B}\right) \sim$ $l_{B}^{-d B}$.

This paper adopts the covering algorithm based on the breadth-first-search to investigate this property. Figure 4 shows the result. According to it, this empirical network has the characteristic of self-similarity with $d B=1$. Roughly speaking, this network is tied together in the same way across increasing levels in its hierarchical organizations, which means the links between clusters of firms, and between clusters of clusters, and so on, obey the same statistical trends as the links between individual firms themselves. In short, the architecture of this firm competition network is symmetrical.

\subsection{Effect of Structural Properties}

In the part, we give some discussions about the effect of structural properties on dynamical processes on networked systems. As mentioned above, the small world effect has been found in this empirical network. It is well known that the small world effect has obvious implications for the dynamics of processes taking place on networks. For example, if one considers the spread of information, or anything else across a network, the small world effect implies that the spread will be fast. In this

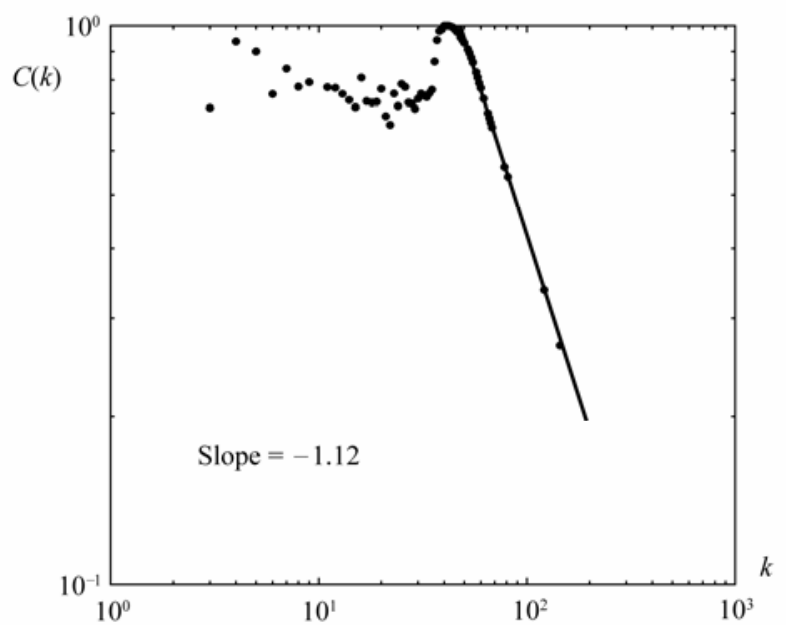

Figure 3. Average clustering coefficient of vertices.

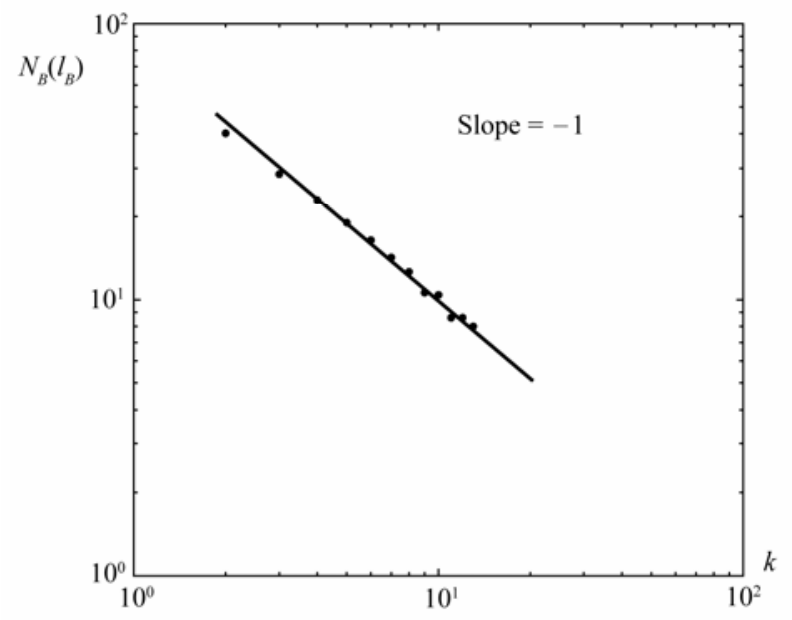

Figure 4. Demonstration of self-similarity.

firm competition network, that means any information with respect to the competitors or any competition fluctuations will spread very fast through the networks.

Moreover, high clustering and positive degree correlation are highly distinctive statistical signatures common to social networks, different from other types of networks. Reference [15] conjectured that the fact that social networks are usually divided into groups or communities is the appropriate explanation for both of these properties. The characteristic of degree correlation has been suggested to affect the resilience to damage of networks [17] and the diffusion of innovations on networks [7]. Compared with networks that are disassortative, it is not surprising that the size of the giant components is smaller in the assortative mixed networks since percolation will be restricted to the sub-network. As for the competition network, it is not clear yet how this structural property 
affects the competitive dynamics on it.

As for power-law degree distribution, the probability of high degree vertices decays not so rapidly, compared with the case with exponential degree distribution. It means that there indeed exist a certain number of extremely high degree vertices, so-called hub vertices. Related to degree distribution is the property of resilience of networks. In literature from different disciplines so far, the importance of hub vertices has been mentioned frequently. It has been observed that many networks with power-law degree distribution are robust against random vertex removal, but less robust to targeted removal of the highest degree vertices. In this firm competition network, the importance of high-degree firms is highlighted therefore, and the removal or any change of these types of firms will have significant effects on the whole network.

There is little attention having been given to the effect of hierarchical modularity and self-similarity on dynamical behaviors of networked systems yet. Reference [15] have proposed that the presence of hierarchical modularity reinterprets the role of the hubs in complex networks, which are known to play a key role of increasing robustness and spreading viruses for scale-free networks. In this firm competition network, the positions of high-degree vertices, which full the structural holes among communities, obviously have some important implications for information spread. For self-similarity, reference [18] has proposed that it can be used as a benchmark for testing models of network structure, which therefore is important to the research on dynamic processes based on the network structural model.

\section{Theoretical Model}

\subsection{Model of the Firm Competition Network}

As mentioned above, the focus of network model research is shifting away from the analysis of single structural properties to consideration of multiple properties of networks. To the best of our knowledge, there is no existing network model that can theoretically reproduce four characteristics of the competitive network simultaneously although they are proved to emerge at the same time. Therefore there is a requirement to propose a theoretical model that can reproduce them at the same time and predict the topology of firm competition network successfully, which will full the gap of existing models to some extent.

As mentioned in the part of introduction, competition behaviors are omnipresent behaviors, which could be found everywhere. We found many valuable literatures in the research field of competitive food web, studying the structural properties of food webs and proposing several theoretical network models [19,20]. Between the competitions among species and the competitions among firms, there indeed exists some essential similarity. Drawing valuable ideas from this completely different discipline, we form and describe our theoretical network model as follows.

In fact, in firm competition networks, the features of its developed products, such as the product function or the customer target, can characterize each firm. And an edge between two firms is constructed if their characteristics are similar and thus two firms form competitive relationship. It hints two possible factors affecting the topology of the network. First is heterogeneity in vertices characteristics. In fact, if all vertices characteristics are completely homogeneous, that is, all firms in the real system (such as in an industry or a park) produce the similar products, then the corresponding network is fully connected, which is not discussed in this paper. On the other hand, in view of products features, there is a competing range for each firm, for instance in reality the field of e-commerce application platform or the field of enterprise management system. All firms in the system whose developed products have the features falling in this range are the competitors of this firm due to the similarity in their products features. Other firms out of this range have no direct competition with this firm. The ranges vary depending on different products developed by firms, which accordingly affect the connection of network.

Therefore, the theoretical model can be constructed as follows. Given $N$ vertices, each vertex $i$ is assigned a characteristic value $x_{i}$ that is drawn from a beta distribution $\operatorname{Beta}(1, \alpha)$ where $\alpha \in(1,+\infty)$ quantifies the heterogeneity level in nodes' characteristic values. Concretely, the heterogeneity level is increasing as $\alpha$ decreases towards 1. Especially, when $\alpha=1$ all nodes' characteristic values are drawn from the uniform distribution. As mentioned above, in view of some practical limits, the vertices would be constrained to compete with vertices within a certain range. Expressed by formula, if $\left|\left(x_{i}-x_{j}\right)\right| \leq\left(r_{i}+r_{j}\right) / 2$ then vertex $i$ and $j$ are connected, or else two vertices are not connected. Here, $r_{i}$ is assumed to be a random variable from a beta distribution $\operatorname{Beta}(1, \sigma)$ where $\sigma$ represents the variability of $r_{i}$. It is known that $E(x)=1 /(1+\alpha)$ for $x \sim \operatorname{Beta}(1, \alpha)$ and $E(r)=1 /(1+\sigma)$ for $r \sim \operatorname{Beta}(1, \sigma)$. In order to assure the model-generated network's average connectivity close to the empirical network's, we choose $\alpha$ and $\sigma$ in simulation to satisfy $1 /(1+\alpha)(1+\sigma)=C / 4$ where the left item is just $E(x) \cdot E(r)$ and $C$ equals $2 L / N(N-1)$ that is just the network density being the ratio between the number $L$ of actual links and the possible number $N(N-1) / 2$ in the empirical undirected network. By adjusting the parameter $\alpha$ and $\sigma$, we can find out the optimal network that predicts those empirical characteristics better than all other model-generated networks. 


\subsection{Analysis of Simulation Results}

In our simulation, according to the empirical firm competition network, the parameters $N$ and $C$ in the model are respectively 574 and 0.04 . In order to obtain the optimal predicted network, $\alpha$ is chosen to be 1.5 and then $\sigma$ can be calculated by solving $1 / 2.5(1+\sigma)=0.04$.

Figure 5 presents the comparison of structural properties between the empirical network and the model-generated network. The value in the bracket is the slope of corresponding solid line. As it indicates, our proposed model generates the network displaying multiple properties simultaneously, including power-law degree distribution, hierarchical modularity, positive degree correlation and self-similarity, as similar as the empirical network. Meanwhile, all four properties are predicted successfully. Especially, as shown in the plot (a), the full predicted data of degree distribution displays good fit with the empirical data. Therefore, this simple network model has the power to provide mechanistic explanations for the structural complexity of firm competition networks.

\section{Concluding Remarks}

In summary, this paper has thrown some empirical and
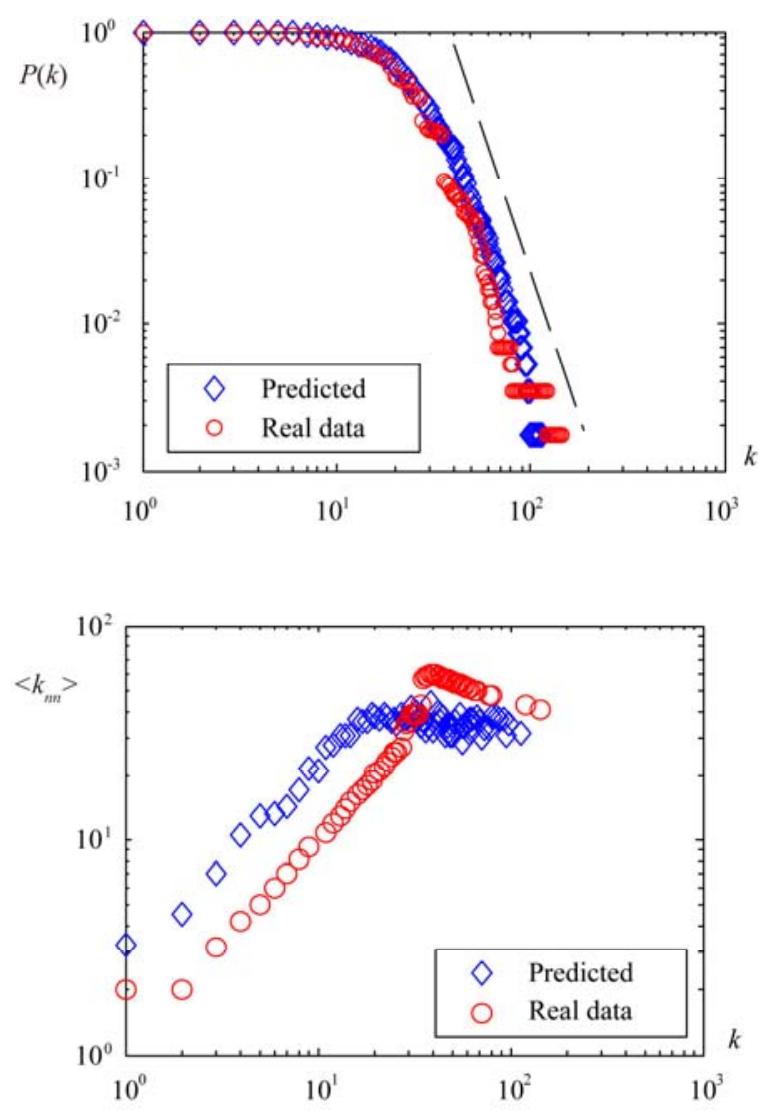

theoretical insights into the topological complexity of firm competition networks. In looking forward to future directions in this area, it is clear that there is much to be done.

Firstly, the structure analysis of firm competition networks is only the first step. In a sense, our ultimate goal is to understand the behaviors and functions of this special competition networks. Therefore, the question to be explored next is how these observed structural properties affect the competitive dynamics on earth. So far, researchers from different disciplines have developed a variety of techniques and models, helping us understand or predict the behaviors of networked system, however, studies of the effect of structure on system behavior are still in their infancy. The next thing we need to do is to study the effect of structural properties mentioned in this paper on the competitive dynamics of firm competition networks.

In addition, although the theoretical model proposed in this paper could reproduce the topology of the firm competition network to some extents, there are many levels of sophistication one can add to this model to make it more appropriate for real competitive networks. For example, the model should not be static, but may
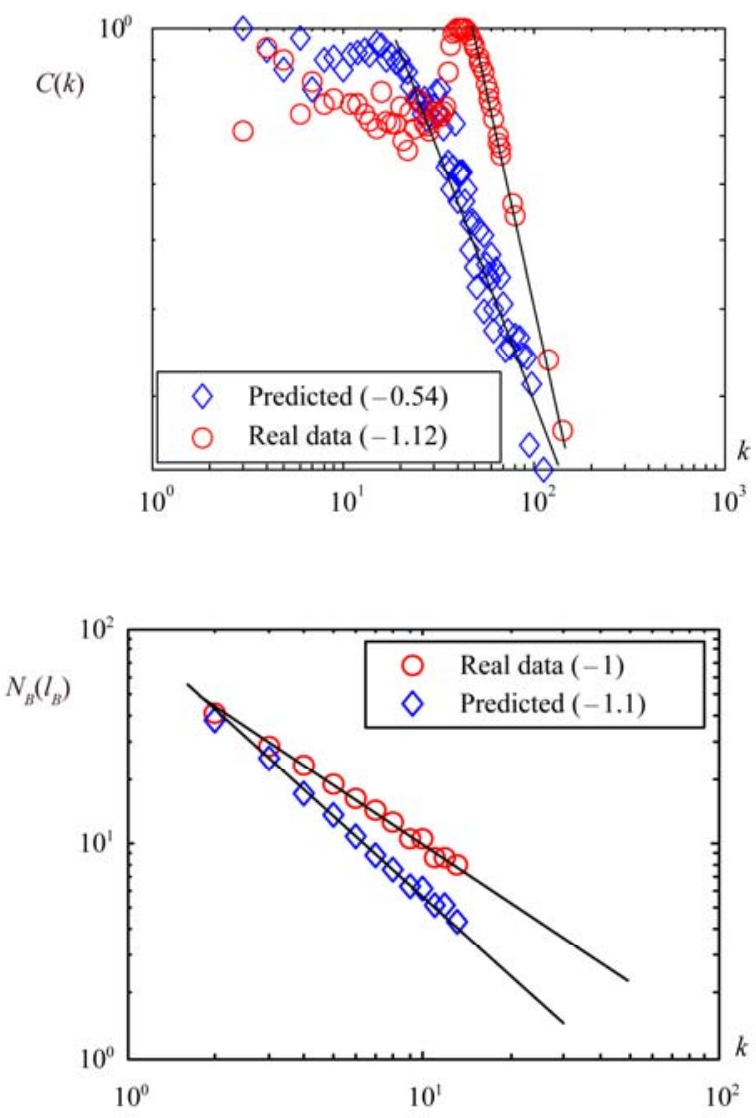

Figure 5. Comparison of the empirical network (red circle) and the model-generated network (blue diamond). 
evolve over time with vertices or edges appearing or disappearing, or values defined on them changing. Moreover, in the future research, we can use this theoretical model as a research platform to explore a vast variety of complex and poorly understood competitive phenomena in the field of industry organization. For example, how do the competition networks evolve? How does the different position in the networks influence the individual firm's control ability of competition? How do the fluctuations spread on the competition networks?

It is worthy to note that although the research in this paper is constructed on the basis of firm competition networks, it may be extended to the analysis of the general competition systems. As mentioned above, competitive phenomena are omnipresent in real socio-economical systems. We hope that our preliminary investigations in the firm competition will stimulate other researchers to pursue more extensions.

\section{Acknowledgements}

The authors would like to thank CHEN Xiao-rong for her fruitful discussions during the development of this paper.

\section{REFERENCES}

[1] M. E. J. Newman, "The Structure and Function of Complex Networks," SIAM Review, Vol. 45, No. 2, 2003, pp. 167-256. doi:10.1137/S003614450342480

[2] R. Albert and A.-L. Barabasi, "Statistical Mechanics of Complex Networks," Reviews of Modern Physics, Vol. 74, No. 1, 2002, pp. 47-97. doi:10.1103/RevModPhys.74.47

[3] D. J. Watts, "The "New" Science of Networks," Annual Review of Sociology, Vol. 30, 2004, pp. 243-270. doi:10.1146/annurev.soc.30.020404.104342

[4] M. E. J. Newman, "The Structure of Scientific Collaboration Networks", Proceedings of the National Academy of Sciences, USA, Vol. 98, No. 2, 2001, pp. 404-409. doi: $10.1073 /$ pnas. 021544898

[5] M. Riccaboni and F. Pammolli, "On Firm Growth in Networks," Research Policy, Vol. 31, No. 8-9, 2002, pp. 1405-1416. doi:10.1016/S0048-7333(02)00071-9

[6] M. Parhi, "Dynamics of Inter-Firm Linkages in Indian Auto Component Industry: A Social Network Analysis," DRUID Winter Conference, Denmark, January 2005.

[7] X. Guardiola, A. Diaz-Guilera, C. J. Perez, A. Arenas and M. Llas, "Modelling Diffusion of Innovations in a Social
Network," Physical Review E, Vol. 66, No. 2, 2002. doi:10.1103/PhysRevE.66.026121

[8] A. Arenas, A. Daz-Guilera, C. J. Prez and F. VegaRedondo, "Self-Organized Evolution in Socio-Economic Environments," Physical Review E, Vol. 61, No. 4, 2000, pp. 3466-3469. doi:10.1103/PhysRevE.61.3466

[9] D. R. Gnyawali and R. Madhavan, "Cooperative Networks and Competitive Dynamics: A Structural Embeddedness Perspective," Academy of Management review, Vol. 26, No. 3, 2001, pp. 431-445. doi:10.2307/259186

[10] B. Uzzi, "Social Structure and Competition in Interfirm Networks: The Paradox of Embeddedness," Administrative Science Quarterly, Vol. 42, No. 1, 1997, pp. 35-67. doi: $10.2307 / 2393808$

[11] M. Granovetter, "Economic Action and Social Structure: The Problem of Embeddedness," American Journal of Sociology, Vol. 91, No. 3, 1985, pp. 481-510. doi:10.1086/228311

[12] A.-L. Barabasi and R. Albert, "Emergence of Scaling in Random Networks." Science, Vol. 286, No. 5439, 1999, pp. 509-512. doi:10.1126/science.286.5439.509

[13] E. Ravasz and A.-L. Barabasi, "Hierarchical Organization in Complex Networks," Physical Review E, Vol. 67, No. 2, 2003, pp. 026112-1-7. doi:10.1103/PhysRevE.67.026112

[14] A. Vazquez, "Growing Network with Local Rules: Preferential Attachment, Clustering Hierarchy, and Degree Correlations," Physical Review E, Vol. 67, No. 5, 2003, 056104. doi:10.1103/PhysRevE.67.056104

[15] M. E. J. Newman and J. Park, "Why Social Networks are Different from Other Types of Networks," Physical Review E, Vol. 68, No. 3, 2003, 036112.

[16] C. Song, S. Havlin and H. A. Makse, "Self-Similarity of Complex Networks," Nature, Vol. 433, No. 7024, 2005, pp. 392-395. doi:10.1038/nature03248

[17] A. Vazquez and Y. Moreno, "Resilience to Damage of Graphs with Degree Correlations," Physical Review E, Vol. 67, No. 1, 2003, 015101. doi:10.1103/PhysRevE.67.015101

[18] S. H. Strogatz, "Romanesque Networks," Nature, Vol. 433, No. 7024, 2005, pp. 365-366. doi:10.1038/433365a

[19] R. J. Williams and N. D. Martinez, "Simple Rules Yield Complex Food Webs," Nature, Vol. 404, No. 6774, 2000, pp. 180-183. doi:10.1038/35004572

[20] N. Rooney, K. S. McCann and J. C. Moore, "A Landscape Theory for Food Web Architecture," Ecology Letters, Vol. 11, No. 8, 2008, pp. 867-881. doi:10.1111/j.1461-0248.2008.01193.x 